\title{
INCONTINENCIA URINARIA Y CALIDAD DE VIDA EN MUJERES JÓVENES DE UN CENTRO DE SALUD FAMILIAR EN SANTIAGO DE CHILE
}

Urinary Incontinence and Quality of Life in Young Women in a Family Health Center in Santiago de Chile

Pascuala Alvarado Briceño ${ }^{1}$, Hilda Bonilla`Gómez ${ }^{2}$, Rodrigo Asun ${ }^{3}$

Autora correspondencia: Pascuala Alvarado Briceño

Correo electrónico: pazalvarado@gmail.com

1. Magister en Salud de la Mujer. Matrona-Docente Escuela de Obstetricia y Puericultura, Universidad Mayor. (Santiago, Chile)

2. Magister en Salud Pública. Matrona. Docente Escuela de Obstetricia y Puericultura, Universidad Mayor. (Santiago, Chile)

3. Director de carrera de Sociología, Universidad de Chile, Santiago, Chile. (Santiago, Chile)

Fecha de recepción: 19/08/2020 Fecha de aceptación: 03/06/2020

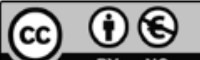




\section{RESUMEN}

OBJETIVO. Determinar asociación entre Calidad de Vida (CV) e Incontinencia Urinaria (IU) en mujeres de 20 a 44 años del Centro de Salud Familiar Santa Julia, Santiago de Chile. MATERIALES Y MÉTODOS. Estudio analítico de corte transversal realizado desde septiembre a diciembre de 2018. De 661 mujeres encuestadas, 184 refirieron IU, de estas, 170 participaron en el estudio. Valor media de edad: 33,84 años. Para la IU se aplicó la Escala de Sandvik y para la CV y la CVRS, se aplicaron los instrumentos ICIQ- SF y el de Potenziani, respectivamente. RESULTADOS. La prevalencia de IU fue 27,8 \%. La severidad de la IU fue moderada en el 55,9 \%, grave en un $12,3 \%$ y muy grave en un $1,8 \%$. Según el ICIQ-SF el impacto de la IU en la CV de moderado a muy intenso, fue de $65,9 \%$ (18,8 \% moderado, $22,9 \%$ intenso y $24,2 \%$ muy intenso). Según el cuestionario de Potenziani, 1 de cada 4 mujeres del estudio percibe una repercusión intensa en su CV. Se aplicó Chi Cuadrado y Coeficiente V de Cramer observando correlación positiva moderada y estadísticamente significativa de 0,419 entre severidad IU y CV. Además, correlación positiva moderada y estadísticamente significativa de 0,463 entre el impacto percibido por las mujeres y la repercusión en su CV. CONCLUSIÓN. La IU afecta la CV de las mujeres desde edades tempranas y aumenta con los años. Es fundamental diagnosticar precozmente, generar políticas públicas sanitarias e implementar estrategias locales para tratar esta patología biopsicosocial.

Palabras clave: Calidad de vida; Incontinencia Urinaria; Salud de la mujer.

\section{ABSTRACT}

AIM. To determine the association between Quality of Life (QOL) and Urinary Incontinence (UI) in women of ages between 20 and 44 from the Santa Julia Family Health Center, Santiago de Chile. MATERIALS AND METHODS. Analytical cross-sectional study carried out from September to December 2018. Of 661 women surveyed, 184 referred UI, of these, 170 participated in the study. Average age value 33.84 years. For UI, the SANDVIK Scale was applied and for QoL and HRQoL, the ICIQ-SF and Potenziani instruments were applied, respectively. RESULTS. The prevalence of UI was $27.8 \%$. The severity of the UI was moderate in $55.9 \%$, severe in $12.3 \%$ and very severe in $1.8 \%$. According to the ICIQ-SF, the impact of UI on QoL from moderate to very intense was $65.9 \%$ (18.8\% moderate, $22.9 \%$ intense and $24.2 \%$ very intense). According to the POTENZIANI questionnaire, 1 in 4 women in the study perceives an intense impact on their QoL. Chi Square and Cramer's V Coefficient were applied, observing a moderate and statistically significant positive correlation of 0.419 between UI and CV severity. In addition, a moderate and statistically significant positive correlation of 0.463 between the impact perceived by women and the impact on their QoL. CoNCLUSIONS. UI affects women's QoL from an early age and increases with age. It is essential to diagnose early, generate public health policies and implement local strategies to treat this biopsychosocial pathology.

Keywords: Quality of Life; Woman's health; Urinary Incontinence. 


\section{INTRODUCCIÓN}

La «International Continence Society» (ICS) define la Incontinencia urinaria (IU) como «la pérdida involuntaria de orina objetivamente demostrable que origina un problema social o higiénico» (Abrams et al., 1988, p. 5). En ese sentido, se ha observado una relación directa entre la IU y la Calidad de Vida $(\mathrm{CV})$, producto del deterioro del bienestar de las mujeres, reduciendo su autonomía y autoestima (Higa et al., 2008). En ese sentido, la $\mathrm{CV}$ es entendida como la percepción del individuo sobre su posición en la vida dentro del contexto cultural y el sistema de valores en el que vive con respecto a sus metas, expectativas, normas y preocupaciones (OMS, 1994). Existen diversas definiciones sobre la CV. Felce \& Perry (1995) proponen cuatro conceptualizaciones:

1) Como la calidad de las condiciones de vida de una persona; 2) La satisfacción experimentada por la persona con dichas condiciones vitales; 3) La combinación de componentes objetivos y subjetivos, es decir, calidad de vida definida como la calidad de las condiciones de vida de una persona junto a la satisfacción que ésta experimenta; 4) La combinación de las condiciones de vida y la satisfacción personal ponderadas por la escala de valores, aspiraciones y expectativas personales. (pp. 51-74)

Específicamente, la Calidad de Vida Relacionada con la Salud (CVRS) es concebida como la capacidad del individuo para realizar las actividades importantes tanto en el aspecto afectivo, social y funcional, siendo aquello influenciado por la percepción subjetiva del individuo (Mejía et al., 2007). De este modo, la CV es inherente al ser humano, pues se relaciona con el grado de satisfacción de la persona con su situación física, su estado emocional, su vida afectiva familiar, amorosa y social, considerando el sentido que le atribuya a la misma. Al profundizar en la relación entre Calidad de Vida e Incontinencia Urinaria, se observa un inicio precoz (antes de los 45 años) identificándose mayores dificultades, sobre todo, al realizar actividades físicas, laborales y sociales. En esa línea, un estudio realizado en la Universidad de Navarra en España señaló que la prevalencia de IU en mujeres adultas varía entre un $20 \%$ a un $50 \%$ y en adultas jóvenes en un $20 \%$ a $30 \%$ (Robles, 2006). Por su parte, el estudio de Rebassa et al. (2013) identificó que un $24 \%$ de las mujeres sufrían IU y que el 25,2 \% que padecía IU de moderada a severa presentaba score de ICIQ-SF ${ }^{1}$ de 9.2 ; que las mujeres que presentaban

1 El «International Consultation on Incontinence Questionnaire Short-Form» (ICIQ-SF) es un instrumento que evalúa la Incontinencia Urinaria en las mujeres, es un cuestionario breve dirigido a medir la percepción de los síntomas de la IU y la calidad de vida de las usuarias (Busquets, et al., 2012). 
IU mixta o continua tenían score ICIQ-SF más alto, a la vez que mostraron peor CV en todas las dimensiones del instrumento EuroQol-5D. ${ }^{2}$

Desde la dimensión psicosocial, Higa et al. (2008) afirman que las mujeres menores de 50 años ocultan la IU por temor al rechazo social, concepción de envejecimiento, vergüenza y temor a pérdida de orina en público o durante la relación sexual. En concordancia, el estudio de Modroño et al. (2004) detectó una baja tasa de consulta, de un $43,2 \%$, esto, principalmente ocasionado por la vergüenza que genera en las mujeres asumir la pérdida de orina como un problema de salud.

De este modo, la IU afecta el bienestar psicológico, social y físico de las mujeres restringiendo su estilo de vida. La prevención e identificación de la etiología de la IU junto a un plan de tratamiento individualizado son esenciales para mejorar la CV (ACOG, 2015).

No obstante, en Chile no existen suficientes estudios que relacionen la IU con impacto en la CV de las mujeres de 20 a 44 años. Dentro de las pocas investigaciones nacionales se encuentran el estudio realizado por Salazar et al. (2005), quienes encontraron una prevalencia de $62,2 \%$ en mujeres mayores de 30 años con un promedio de edad de 52,8 años. El estudio realizado por Harwardth et al. (2004), quienes hallaron una prevalencia de 77,1 \% en mujeres de 30 a 84 años, y, por último, el estudio de Rincón (2015), quien describe una prevalencia de $62,6 \%$ en mujeres entre 30 y 81 años.

Según lo expuesto, se trata de un problema subdiagnosticado. En Chile no existen estrategias de prevención en el Servicio Público de Salud, solo tratamientos para los casos más severos de IU. No obstante, su atención no es prioritaria, puesto que no es una patología incluida dentro del Programa de Garantías Explícitas en Salud (GES), Ley 19.966 (2004), lo cual demora la atención de especialidad y su resolución. Por este motivo, nuestro objetivo fue determinar la asociación entre Calidad de Vida (CV) e Incontinencia Urinaria (IU) en mujeres de 20 a 44 años del Centro de Salud Familiar (CESFAM) Santa Julia, Santiago de Chile.

\footnotetext{
${ }^{2}$ EuroQol-5D o en su forma abreviada EQ-5D, es un instrumento genérico de medición de la Calidad de Vida Relacionada con la Salud (CVRS), se utiliza en pacientes sanos como en los que presentan alguna patología (Herdman et al., 2001).
} 


\section{MATERIAL Y MÉTODO}

Esta investigación corresponde a un diseño observacional, cuantitativo, transversal y descriptivo correlacional que mide la magnitud de un problema de salud (IU) y su influencia en la CV de las mujeres afectadas, mediante la aplicación de cuatro test de respuestas cerradas.

El universo del estudio fueron 170 mujeres de 20 a 44 años pertenecientes al Centro de Salud Familiar (CESFAM) Santa Julia, ubicado en Santiago de Chile. El estudio se realizó durante los meses de septiembre a diciembre de 2018.

Inicialmente, se aplicó una encuesta elaborada por el investigador principal (primer autor) con el único objetivo de indagar la presencia de IU (ver Anexo 1). Se preguntó a un total de 661 mujeres, entre 20 y 44 años respecto de la presencia de IU. De estas, 184 refirieron presentar algún grado de incontinencia.

Luego de aplicar los siguientes criterios de exclusión:

- Mujeres con sintomatología sugerente de infección urinaria

- Mujeres embarazadas

- Mujeres con déficit de lectoescritura, discapacidad cognitiva o barrera idiomática

Se seleccionó un total de 170 mujeres a las cuales se les informó respecto de la voluntariedad de la participación. De estas, todas aceptaron participar en el estudio previa firma del Consentimiento Informado. Cabe destacar que este estudio cuenta con la aprobación del Comité Ético Científico de la Universidad Mayor de Chile.

Las 170 participantes contestaron, en primer lugar, la encuesta de Sandvik (1993) (ver Anexo 2), la cual mide el grado de severidad de la IU, luego, la encuesta ICIQ-SF (ver Anexo 3), la cual vincula el grado de severidad y la CV definiendo el tipo de incontinencia según sintomatología. Finalmente, se aplicó la escala de Potenziani (2005), que evalúa la repercusión de la IU en la CVRS de las mujeres (ver Anexo 4).

Los datos fueron vaciados en una hoja de cálculo MS Excel y analizados con el programa estadístico SPSS Statistics ${ }^{\circledR}$ por el segundo investigador de este estudio.

El análisis de variables de naturaleza cualitativa o categórica se realizó a través de medidas de frecuencia (proporciones). La independencia o asociación entre las variables entre sí fue medida mediante el cálculo de Chi cuadrado (se aplicó el Coeficiente V de 
Cramer). En caso de las variables cuantitativas, se estimaron medias y, posteriormente, se realizó el análisis correlacional mediante el cálculo de coeficiente de correlación de Pearson.

El análisis de los datos contempló a) análisis descriptivo por pregunta para todo el grupo de estudio; b) análisis descriptivo de preguntas claves y puntuaciones agregadas por cuestionario para la muestra total y subgrupos de edad; c) caracterización según índice de severidad; y d) análisis de la relación (significación e intensidad) entre preguntas claves del cuestionario ICIQ-SF y su impacto en la CV, según el cuestionario Potenziani para la muestra total y subgrupos de edad. Para evaluar la relación entre las variables frecuencia, magnitud y el impacto en $\mathrm{CV}$ - se aplicó la prueba de Chi cuadrado.

\section{RESULTADOS}

En el grupo estudiado (mujeres de 20 a 44 años) se observó una prevalencia de IU de $27,8 \%$, IU de esfuerzo en el $47,6 \%$, IU de urgencia en un $22,9 \%$, mixta en un $21,2 \%$ y la IU considerada global fue de un $8,2 \%$.

En relación con el grado de impacto en la CV considerando la frecuencia y cantidad de la pérdida de orina, según ICIQ-SF percibido por las mujeres del estudio, se observó en la distribución del grado de impacto un valor medio de $5,44 \pm 3,32$, con un valor mínimo de 0 y un valor máximo de 10 , la mediana obtuvo un valor de 5 . La media del puntaje total de ICIQ-SF con un mínimo de 3 y máximo de 20, fue 10,47 con una DS 4,377 .

Se observa que, del $100 \%$ de las mujeres, 1 de cada 4 entre 20 y 44 años percibe una repercusión intensa en su calidad de vida (ver Figura 1).

Figura 1. Repercusión de la IU en la calidad de vida según escala Potenziani en mujeres de 20 a 44 años del CESFAM Santa Julia, Santiago de Chile

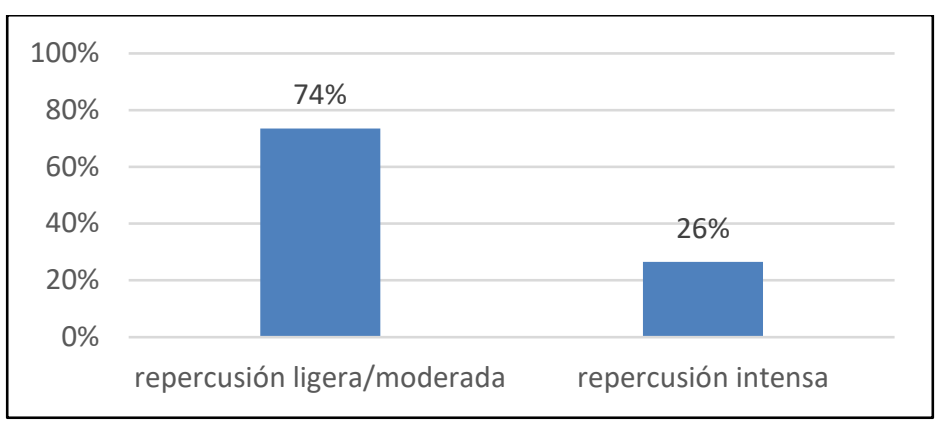


La severidad estimada según la escala de Sandvik muestra que el 50 \% de las mujeres refiere una frecuencia entre algunas veces a la semana a todos los días o noches. Así, un $64,1 \%$ refiere que el escape es de chorro o más. Al analizar la severidad del cuadro, un $14,1 \%$ de las mujeres presenta una severidad grave o muy grave, y el 55,9\% presenta severidad moderada (ver Tabla 1).

Tabla 1. Distribución de frecuencia, cantidad y severidad de IU, según escala de Sandvik en mujeres de 20 a 44 años del CESFAM Santa Julia, Santiago de Chile

\begin{tabular}{|c|c|c|c|}
\hline \multirow[t]{2}{*}{ Parámetro } & & \multicolumn{2}{|c|}{ Frecuencia } \\
\hline & & & Porcentaje \\
\hline \multirow[t]{6}{*}{ Frecuencia } & $<1 \mathrm{v}$ al mes & 28 & 16,5 \\
\hline & Algunas veces al mes & 57 & 33,5 \\
\hline & Algunas veces a la & 58 & 34,1 \\
\hline & Semana & & \\
\hline & Todos los días/noches & 27 & 15,9 \\
\hline & Total & 170 & 100,0 \\
\hline \multirow[t]{4}{*}{ Cantidad } & Gota & 61 & 35,9 \\
\hline & Chorro & 100 & 58,8 \\
\hline & Mucha cantidad & 9 & 5,3 \\
\hline & Total & 170 & 100,0 \\
\hline \multirow[t]{5}{*}{ Severidad } & leve & 51 & 30,0 \\
\hline & moderado & 95 & 55,9 \\
\hline & grave & 21 & 12,3 \\
\hline & muy grave & 3 & 1,8 \\
\hline & Total & 170 & 100,0 \\
\hline
\end{tabular}

Relacionado con la percepción de impacto, se agruparon las respuestas según la intensidad del impacto percibido de acuerdo con la siguiente categorización: No percibe impacto en su calidad de vida (0), impacto leve (1-3), impacto moderado (4-6), impacto intenso (7-9) y muy intenso (10) (Laserre, 2009). Como resultado se obtuvo que al $40 \%$ de las mujeres les impacta en forma intensa o muy intensa, al 25,9 \% les afecta en forma moderada, al $27,1 \%$ en forma leve y a un $7 \%$ no les impacta en su calidad de vida (ver Tabla 2). En consecuencia, se observa en este grupo que, 1-2 de cada 4 mujeres entre 20 a 44 años percibe un impacto intenso en su calidad de vida. 
Tabla 2. Percepción de grado de impacto leve, moderado, intenso y muy intenso en calidad de vida, considerando la frecuencia y cantidad de la pérdida de orina según ICIQ-SF en mujeres de 20 a 44 años del CESFAM Santa Julia, Santiago de Chile

Grado de impacto

No impacta (0)

Leve (1-3)

Moderado (4-6)

Intenso (7-9)

Muy intenso (9 a 10)

Total

A medida que aumenta la severidad de la IU en las mujeres, la proporción de repercusión intensa también se ve aumentada alcanzando un 57,1\% en las mujeres con severidad grave. El coeficiente de correlación indica que existe una correlación positiva moderada entre las variables de 0,419 , siendo ésta, estadísticamente significativa. La relación entre la severidad de la IU medido por escala Sandvik y la repercusión en la calidad de vida medido por la escala Potenziani se observa a continuación (ver Tabla 3 ).

Tabla 3. Relación entre la severidad de la IU medida por escala Sandvik y repercusión en la calidad de vida según la escala Potenziani en mujeres de 20 a 44 años del CESFAM Santa Julia, Santiago de

\section{Chile}

\begin{tabular}{|c|c|c|c|c|c|}
\hline \multirow{2}{*}{\multicolumn{3}{|c|}{$\begin{array}{c}\text { Grado de severidad de IU } \\
\text { Sandvik }\end{array}$}} & \multicolumn{2}{|c|}{ Repercusión Potenziani } & \multirow[t]{2}{*}{ Total } \\
\hline & & & ligera/moderada & intensa & \\
\hline \multirow[t]{8}{*}{ Sandvik } & \multirow[t]{2}{*}{ leve } & $\mathrm{n}$ & 48 & 3 & 51 \\
\hline & & $\%$ & 94,1 & 5,9 & 100 \\
\hline & \multirow[t]{2}{*}{ moderado } & $\mathrm{n}$ & 68 & 27 & 95 \\
\hline & & $\%$ & 71,6 & 28,4 & 100 \\
\hline & \multirow[t]{2}{*}{ grave } & $\mathrm{n}$ & 9 & 12 & 21 \\
\hline & & $\%$ & 42,9 & 57,1 & 100 \\
\hline & \multirow{2}{*}{$\begin{array}{l}\text { muy } \\
\text { grave }\end{array}$} & $\mathrm{n}$ & 0 & 3 & 3 \\
\hline & & $\%$ & 0,0 & 100 & 100 \\
\hline \multirow{2}{*}{\multicolumn{2}{|c|}{ Total }} & $\mathrm{n}$ & 125 & 45 & 170 \\
\hline & & $\%$ & 73,5 & 26,5 & 100 \\
\hline
\end{tabular}


A medida que aumenta el grado de impacto, la proporción de repercusión intensa también aumenta, alcanzando un $58,5 \%$ en las mujeres con impacto muy intenso. El coeficiente de correlación indica que existe una correlación entre las variables de 0,463 , siendo estadísticamente significativa. La relación entre el impacto en la calidad de vida medido por la escala ICIQ-SF y la repercusión en la calidad de vida según la escala Potenziani, se señala en la Tabla 4.

Por cada unidad de cambio en la severidad, el riesgo de presentar una repercusión intensa aumenta en un $62.7 \%$, relación que se evidencia independiente de la edad de las mujeres, pues el modelo de regresión se rige por dicha variable. Además, no se encontró relación entre la edad y la repercusión. La relación existente entre el impacto en la calidad de vida evidenciado en la escala ICIQ-SF y la repercusión en la calidad de vida medido por la escala Potenziani ajustado por edad se describe a continuación (ver Tabla 5).

Tabla 4. Relación entre el impacto percibido en la calidad de vida según escala ICIQ-SF y repercusión en la calidad de vida según la escala Potenziani en mujeres de 20 a 44 años del CESFAM Santa Julia, Santiago de Chile

\begin{tabular}{|c|c|c|c|c|}
\hline \multicolumn{2}{|c|}{ Impacto según ICIQ-SF } & \multicolumn{2}{|c|}{ Repercusión Potenziani } & \multirow{3}{*}{$\begin{array}{r}\text { Total } \\
58\end{array}$} \\
\hline & & \multirow{2}{*}{$\begin{array}{c}\text { Ligera/Moderada } \\
56\end{array}$} & \multirow{2}{*}{$\begin{array}{r}\text { Intensa } \\
2\end{array}$} & \\
\hline Leve (0-3) & $\mathrm{N}$ & & & \\
\hline & $\%$ & 96,6 & 3,4 & 100 \\
\hline \multirow[t]{2}{*}{ Moderado (4-5) } & $\mathrm{N}$ & 29 & 3 & 32 \\
\hline & $\%$ & 90,6 & 9,4 & 100 \\
\hline \multirow[t]{2}{*}{ Intenso (6-8) } & $\mathrm{N}$ & 23 & 16 & 39 \\
\hline & $\%$ & 59,0 & 41,0 & 100 \\
\hline \multirow[t]{2}{*}{ Muy Intenso (9-10) } & $\mathrm{N}$ & 17 & 24 & 41 \\
\hline & $\%$ & 41,5 & 58,5 & 100 \\
\hline \multirow[t]{2}{*}{ Total } & $\mathrm{N}$ & 125 & 45 & 170 \\
\hline & $\%$ & 73,5 & 26,5 & 100 \\
\hline
\end{tabular}


Tabla 5. Modelo de regresión para la relación entre la escala ICIQ-SF y la escala Potenziani ajustado por edad en mujeres de 20 a 44 años del CESFAM Santa Julia, Santiago de Chile

\begin{tabular}{|c|c|c|c|c|}
\hline & \multirow[t]{2}{*}{$\operatorname{Exp}(B)$} & \multicolumn{2}{|c|}{$\begin{array}{c}\text { I.C. } 95 \% \text { para } \\
\operatorname{EXP(B)}\end{array}$} & \multirow[t]{2}{*}{ Sig. } \\
\hline & & Inferior & Superior & \\
\hline Edad & ,954 & ,897 & 1,016 &, 141 \\
\hline $\begin{array}{l}\text { Impacto ICIQ- } \\
\text { SF }\end{array}$ & 1,627 & 1,383 & 1,914 &, 000 \\
\hline Constante &, 073 & & & 020 \\
\hline
\end{tabular}

\section{DISCUSIÓN}

En un primer análisis se observó una mayor prevalencia de IU en el grupo de mujeres entre los 30 y 39 años (46,5 \%) a diferencia del estudio de Harward et al. (2004) realizado en Isla de Pascua en Chile, el cual encontró una prevalencia de $25,82 \%$ en el grupo de 30 a 39 años y mayor que el estudio de Buckley \& Lapitan (2010), que encontró una prevalencia de 7 a $37 \%$ en mujeres de 20 a 39 años

En este grupo de estudio, el 70,1 \% (medido por escala de Sandvik) presenta un grado de severidad de moderado a muy grave, siendo esto muy relevante por tratarse de mujeres jóvenes en plena actividad sexual, laboral, social y familiar. Estos resultados difieren de los encontrados por Rincón (2015), quien estudió a 181 mujeres con rango de edad más amplio (30 a 60 años y más), y en las cuales encontró IU un 62,8 \% de severidad de moderado a muy grave, observándose que en grupos de mayor edad aumenta la IU a muy severa y severa.

La media del puntaje total de ICIQ-SF (con un mínimo de 3 y máximo de 20) fue de 10,47 con una DS 4,377, difiriendo de los hallazgos encontrados en el estudio de Neygard (2018), el cual encontró una media de 9,36 con una DS 4,9.

En el grupo estudiado, el grado de impacto en la calidad de vida (ICIQ-SF) categorizado muestra que el impacto entre moderado y muy intenso es de 65,9\% y que 
1-2 de cada 4 mujeres les impacta de forma intensa la IU (40 \%). En este sentido, llama a reflexión que, siendo mujeres jóvenes, el impacto es mayor al observado en otros estudios, como el de Laserre (2009), que abarcó 494 mujeres mayores de 18 años, cuyos resultados difieren de los nuestros, pues se encontró que solo un 51,8 \% refirió un impacto en la calidad de vida entre moderado y muy intenso.

La escala de Potenziani fue otro instrumento aplicado en nuestro estudio, el cual mide la repercusión de la incontinencia urinaria en la calidad de vida de las mujeres. En este estudio se observa que 1 de cada 4 mujeres entre los 20 y 44 años percibe una repercusión intensa en su calidad de vida por la IU, situación que, de acuerdo con los estudios de Dellú (2015) y Debu (2015) genera gran alteración en las relaciones personales, ansiedad provocada por la sensación de humedad permanente, angustia de verse expuesta y, en consecuencia, alteración evidente de la calidad de vida.

Por otro lado, se observa que, a medida que aumenta la severidad de la IU (según la escala de Sandvik), la repercusión intensa también aumenta (según la escala de Potenziani), alcanzando un 57,1\% en las mujeres con severidad grave. El coeficiente de correlación indica que existe una correlación entre las variables de 0,419 , siendo esta estadísticamente significativa y fuerte (Coeficiente V de Cramer: 0,419, correlación positiva significativa $\operatorname{sig}<0,01)$.

Al relacionar el impacto percibido por las mujeres en su calidad de vida medido por la escala ICIQ-SF con la repercusión en la calidad de vida medida por la escala de Potenziani, se observa que a medida que aumenta el grado de impacto en las mujeres, la proporción de repercusión intensa también aumenta, alcanzando un 58,5\% en las mujeres con impacto intenso. A su vez, al observar la relación existente entre ICIQ-SF y Potenziani ajustado por edad, se concluye que, por cada unidad de cambio en la severidad, el riesgo de presentar una repercusión intensa aumenta en un 62,7 \%, independientemente de la edad de las mujeres en el grupo estudiado. En ese sentido, nuestra investigación no encontró relación entre la edad y la repercusión de la IU en su calidad de vida. Se debe considerar que los estudios en relación con la edad dan resultados disímiles, como el estudio de Córcales et al. (2008), quienes describen que la repercusión en la calidad de vida es menor en las pacientes más jóvenes y que, a mayor edad, se observa una peor calidad de vida. Así mismo, los estudios de Aoki et al. (2017) y Carusso et al. (2017), los cuales, a diferencia del estudio anterior, encontraron que a menor edad es peor la calidad de vida, concluyendo que, para mejorar la calidad de vida de las mujeres jóvenes que 
sufren incontinencia urinaria, se debe intervenir precozmente para evitar que aumente con la edad como lo aseveran Aoki et al. (2017) y Milsom (1993, 2019).

La principal limitación del estudio fue la cantidad de cuestionarios aplicados a las pacientes, situación que, en ocasiones, pudo haber generado que las mujeres no leyeran con detenimiento las preguntas de los formularios.

\section{CONCLUSIONES}

Los resultados obtenidos dimensionan la repercusión e impacto en la calidad de vida que produce la Incontinencia Urinaria en mujeres jóvenes. Muestran su relevancia y aportan información que permitirá generar estrategias de prevención y tratamiento precoz de esta enfermedad en atención primaria en Chile.

La alta prevalencia en el grupo de mujeres estudiadas cuyo motivo de consulta no era la Incontinencia Urinaria llama la atención. Esto nos permite concluir que la IU es una patología subdiagnosticada por temor, vergüenza o bien asociada a signo de vejez y falta de atractivo, lo cual hace que esta condición de salud se prolongue en el tiempo y no se aborde precozmente.

Otro aspecto que destacar es que, siendo las afectadas mujeres jóvenes, con media de edad de 33,8 años en plena actividad laboral, social y familiar, un alto porcentaje de ellas presentaba un grado de severidad de incontinencia urinaria importante, y 1 de cada 4 de ellas refería que repercutía en forma intensa en su calidad de vida. A partir de esta relación entre severidad de la incontinencia relacionada con la repercusión en la calidad de vida, concluimos que, a medida que aumenta la severidad de la incontinencia de las mujeres, la proporción de repercusión intensa también aumenta alcanzando un 57,1 \% en mujeres con severidad grave.

En cuanto a la relación entre el impacto percibido y repercusión en calidad de vida, concluimos que, a medida que aumenta el grado de impacto percibido por las mujeres, la proporción de repercusión intensa también aumenta alcanzando un 58,5 \% en las mujeres con impacto intenso con una correlación significativa entre las variables. También se concluye que por cada unidad de cambio en la severidad, el riesgo de presentar una repercusión intensa aumenta en una gran proporción $(62,7$ \%). 
Esta investigación abordó el tema de la calidad de vida de las mujeres jóvenes que sufren incontinencia urinaria por considerar que este es el grupo en el cual se debe intervenir precozmente para evitar que la prevalencia aumente con la edad. Asimismo, este estudio determina que la prevalencia es alta y que repercute negativamente en la calidad de vida de las mujeres jóvenes. Del mismo modo, se pretende estimular al profesional matrón/a en la búsqueda dirigida de esta patología, pues son las profesionales que se encuentran más cercanas a la mujer siendo el primer contacto con estos grupos etarios.

\section{AGRADECIMIENTOS}

Agradecimientos a las mujeres que desinteresadamente aportaron la información para el estudio, al Dr. Rubén Espinoza, director del Centro de Salud Familiar Santa Julia Comuna de Macul, Santiago de Chile, y al personal de ese establecimiento, cuya autorización y apoyo brindado permitió la ejecución de esta investigación. 


\section{BIBLIOGRAFÍA}

ABRAMS, Paul, et al. Estandarización de la terminología de la función del tracto urinario inferior: reporte del subcomité de estandarización de la international continence society (ICS). En: Revista Chilena de Urología. 2006, vol. 71, núm. 2, pp. 79-94.

AMERICAN COLLEge of OBSTETRICIANS and GyneCOlOGist (ACOG). Urinary Incontinence in Women. En: Practice Bulletin. 2015, vol. 126, núm. 5: pp. 915-1129.

BuCKLEY, Brian; LAPITAN, Marie. Prevalence of urinary incontinence in men, women, and children-current evidence: findings of the Fourth International Consultation on Incontinence. En: Urology. 2010, vol. 76, núm. 2, pp. 265-270.

BusqueTs, Maritza; SERRA, Ramón. Validación del cuestionario International Consultation on Incontinence Questionnaire Short-Form (ICIQ-SF) en una población chilena usuaria del Fondo Nacional de Salud (FONASA). En: Revista médica de Chile. 2012, vol 140, núm. 3, pp. 340-346.

CARUSO, Salvatore, et al. Effects of Urinary Incontinence Subtypes on Women's Sexual Function and Quality of Life. En: Urology. 2017, vol. 108, pp. 59-64.

DeBUS, George; KÄSTNER, Rocha. Psychosomatic Aspects of Urinary Incontinence in Women. En: Geburtshilfe Frauenheilkunde. 2015, vol. 75, núm. 2, pp. 165-169.

Dellú, Mayra Cecilia. Incontinência urinária no climatério: prevalência, fatores associados e impacto na qualidade de vida. [Tesis doctoral]. Universidad de Sao Paulo: 2015. Disponible en: http://www.teses.usp.br/teses/disponiveis/6/6136/tde19102015-100242/

De MejíA, Beatriz; Merchán, María Eugenia. Calidad de vida relacionada con la salud (CVRS) en adultos mayores de 60 años: una aproximación teórica. En: Revista hacia la promoción de salud. 2007, vol. 12, pp. 11-24.

FElce, David; Perry, Jonathan. Quality of life: its definition and measurement. En: Research in developmental disabilities.1995, vol 16, núm. 1, pp. 51-74.

GobIERNo DE CHILE. Ley N¹9.966 Establece un Régimen de Garantías Explícitas en Salud. Fue promulgada el 25 de agosto de 2004 y publicada el 03 de septiembre de 2004. Disponible en: www.supersalud.gob.cl/normativa/668/w3article-554.html

HARWARDT, Tamara, et al. Estudio de prevalencia de incontinencia urinaria en mujeres de Isla de Pascua. En: Revista Chilena de Urología. 2004, vol. 69, núm. 1, pp. 29-34.

HASLAM, Jeanette. Incontinencia urinaria: por qué las mujeres no piden ayuda. En: Nurs Times. 2005, vol. 101, pp. 47-48.

Herdman, Michael; BADIA, Ximena; BERRA, Silvina. El EuroQol-5D: una alternativa sencilla para la medición de la calidad de vida relacionada con la salud de atención primaria. En: Atención primaria. 2001, vol. 28, pp. 425-429. 
Higa, Rosângela; Baena de Mores, María Helena; Ribeiro, Lopes. Psychocultural meanings of urinary incontinence in women: a review. En: Revista latino-americana de enfermagem. 2008, vol. 16, núm. 4, pp. 779-786.

LASERRE, Andrea, et al. Urinary incontinence in French women: prevalence, risk factors, and impact on quality of life. En: European urology. 2009, vol. 56, núm. 1, pp. 177183.

MARTínez CórCOLES, Benjamín, et al. Calidad de vida en las pacientes con incontinencia urinaria. En: Actas Urológicas Españolas. 2008, vol. 32, núm. 2, pp. 202-210

Milsom, Ian; GyHagen, María. The prevalence of urinary incontinence. En: Climacteric. 2019, vol. 22, núm. 3, pp. 217-222.

Modroño, María José, et al. Estudio de prevalencia de incontinencia urinaria en mujeres de 18 a 65 años y su influencia en la calidad de vida. Atención primaria, 2004, vol. 34, no 3, p. 134-139

NyGAARD, Christiana Campani, et al. Incontinence and Quality of Life in Female Patients with Obesity. En: Rev Bras Ginecol Obstet. 2018, vol. 40, núm. 9, pp. 534-539.

Pascual, María Ángeles. Estudio epidemiológico EPINCONT. Prevalencia y calidad de vida. Progresos de Obstetricia y Ginecología. 2002, vol. 45, pp. 2-9.

Potenziani, Julio.; PotenZIAni, Silvia. Cuestionarios de validación diagnóstica para diversas patologías urológicas. En: Rev Centro Med. 2005, vol. 50, núm. 1.

REBASSA, Miguel, et al. Incontinencia urinaria en mujeres de Mallorca: prevalencia y calidad de vida. En: Actas Urológicas Españolas. 2013, vol. 37, núm. 6, pp. 354-361.

RINCÓN ARDILA, Olga. Caracterización clínica de la incontinencia urinaria y factores asociados en usuarias de la Unidad de la Mujer del Centro de Salud Familiar Ultraestación en la ciudad de Chillán, Chile. En: Revista médica de Chile. 2015, vol. 143, núm. 2, pp. 203-212

Robles, José Enrique. La incontinencia urinaria. En: Anales del sistema sanitario de Navarra. Gobierno de Navarra, Departamento de Salud: 2006, pp. 219-231.

RORVEIT, Guri, et al. Vaginal delivery parameters and urinary incontinence: The Norwegian EPINCONT study. En: American Journal of Obstetrics \& Gynecology. 2003, vol. 189, núm. 5, pp. 1268-1274.

SALAZAR, Aníbal, et al. Prevalencia y factores de riesgo de la incontinencia de orina. En: Revista Chilena de Urología. 2005, pp. 55-63.

SANDVIK, Hogne, et al. Validation of a severity index in female urinary incontinence and its implementation in an epidemiological survey. En: Journal of Epidemiology \& Community Health. 1993, vol. 47, núm. 6, p. 497-499.

Silva, María Antonieta. Efectos de la incontinencia urinaria en la calidad de vida de la mujer climatérica. En: Revista Cubana de Obstetricia y Ginecología. 2018, vol. 44 núm. 1, pp. 1-14. 
TEMmL, Christian. Incontinencia urinaria en ambos sexos: tasas de prevalencia e impacto en la calidad de vida y la vida sexual. En: Neurourol Urodyn. 2000, vol. 19, pp. 25971.

UrzúA, Alfonso; CAQUeO-URíZAR, Alejandra. Calidad de vida: Una revisión teórica del concepto. En: Terapia psicológica, 2012, vol. 30, núm. 1, pp. 61-71. 


\title{
ANEXO 1. ENCUESTA ELABORADA POR EL INVESTIGADOR PRINCIPAL
}

\author{
Encuesta sobre existencia de Incontinencia Urinaria \\ Estimada usuaria, con el fin de saber cómo afecta la pérdida de orina involuntaria \\ (Incontinencia Urinaria) en la vida de las personas; deseamos que conteste las siguientes \\ preguntas: \\ ¿Ha presentado alguna pérdida o escape orina en el último mes? (Encierre en un \\ círculo la respuesta) \\ SÍ NO \\ Si su respuesta es SÍ: ¿desea responder algunas preguntas? (Encierre en un círculo \\ la respuesta) \\ SÍ NO \\ Muchas Gracias
}

\section{ANEXO 2. ESCALA DE SANDVICK}

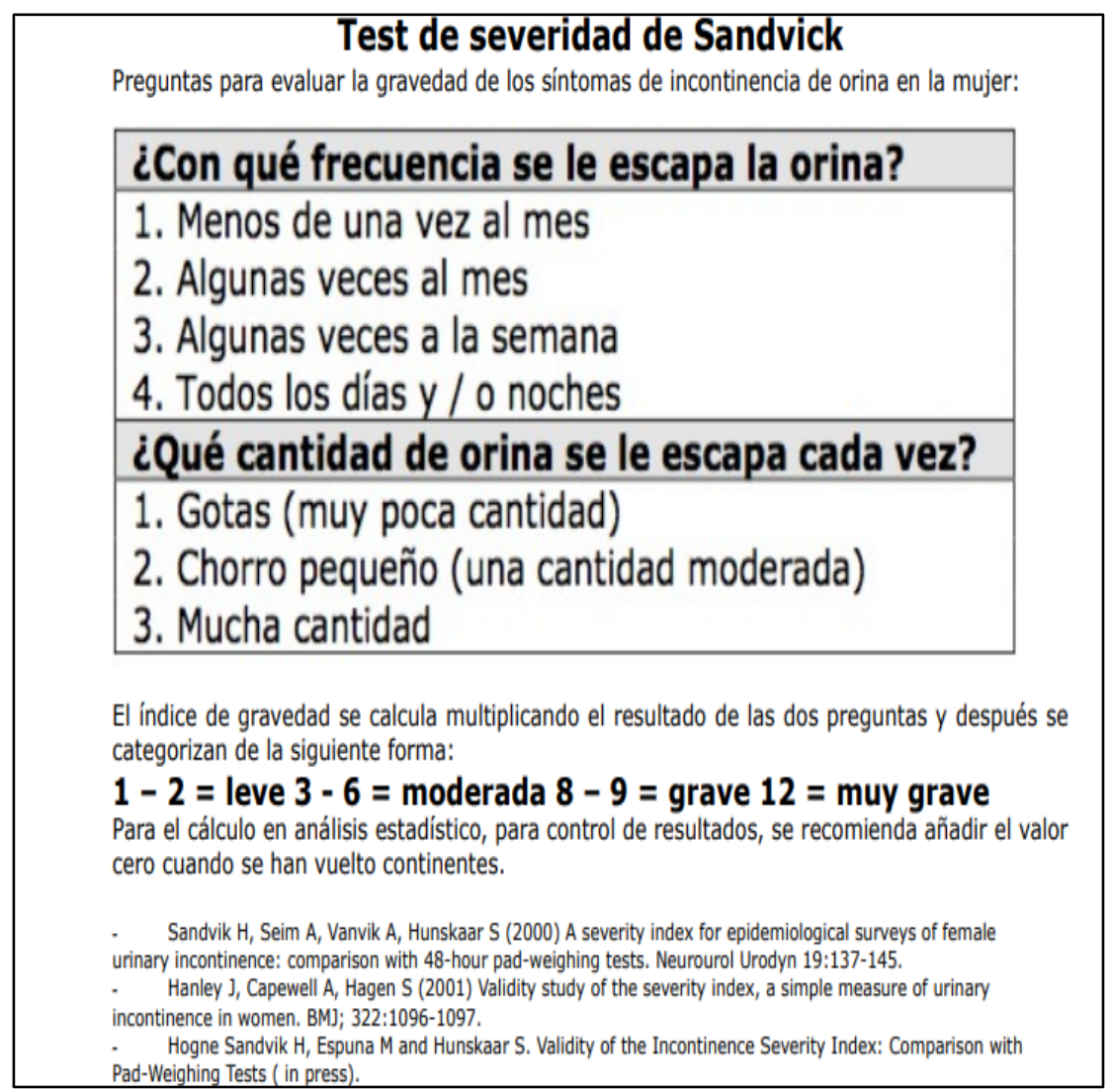




\section{ANEXO 3. ESCALA ICIQ-SF}

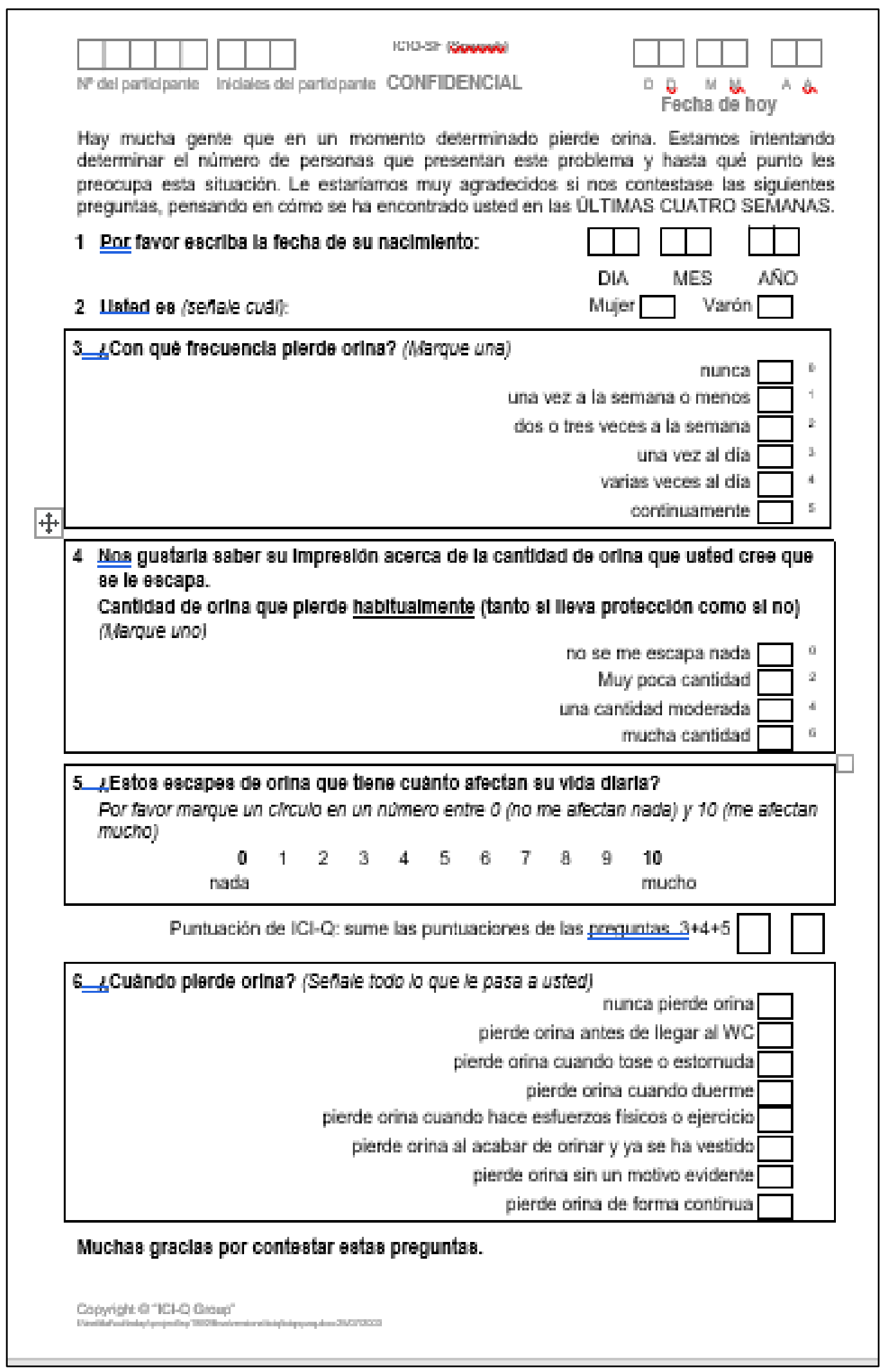




\section{ANEXO 4. ESCALA DE POTENZIANI}

\section{Cuestionario de impacto de la incontinencia urinaria en la calidad de vida de la mujer (Potenziani-14-CI-IO-QOL2000)}

Sencillo cuestionario auto administrado que informa sobre el impacto de la incontinencia en la vida cotidiana de la paciente. Es específico para la incontinencia urinaria en mujeres.

\section{Puntuación final:}

Repercusión LIGERA-MODERADA sobre su calidad de vida=0-14 puntos.

Repercusión INTENSA sobre su calidad de vida= 15-28 puntos.

\begin{tabular}{|c|c|c|}
\hline \multicolumn{3}{|c|}{ 1. ¿Presenta Incontinencia Urinaria en reposo, acostada o sentada? } \\
\hline Nunca & $\mathbf{0}$ & \multirow[t]{3}{*}{ puntos } \\
\hline A veces & 1 & \\
\hline Siempre & 2 & \\
\hline \multicolumn{3}{|c|}{ 2. ¿Presenta Incontinencia Urinaria mientras tose, estornuda o se ríe? } \\
\hline Nunca & $\mathbf{0}$ & \multirow[t]{3}{*}{ puntos } \\
\hline A veces & 1 & \\
\hline Siempre & 2 & \\
\hline \multicolumn{3}{|c|}{ 3. ¿Presenta Incontinencia Urinaria mientras hace esfuerzos, ejercicios aeróbicos o con multifuerza? } \\
\hline Nunca & $\mathbf{0}$ & \multirow[t]{3}{*}{ puntos } \\
\hline A veces & 1 & \\
\hline Siempre & 2 & \\
\hline \multicolumn{3}{|c|}{ 4. ¿Presenta Incontinencia Urinaria mientras realiza su actividad sexual? } \\
\hline Nunca & $\mathbf{0}$ & \multirow[t]{3}{*}{ puntos } \\
\hline A veces & 1 & \\
\hline Siempre & 2 & \\
\hline \multicolumn{3}{|c|}{ 5. ¿Presenta Incontinencia Urinaria relacionada con momentos de urgencia urinaria? } \\
\hline Nunca & $\mathbf{0}$ & \multirow[t]{3}{*}{ puntos } \\
\hline A veces & 1 & \\
\hline Siempre & 2 & \\
\hline \multicolumn{3}{|c|}{ 6. ¿Presenta Incontinencia Urinaria con la vejiga llena o inclusive con la vejiga con poca orina? } \\
\hline Nunca & $\mathbf{0}$ & \multirow[t]{3}{*}{ puntos } \\
\hline A veces & 1 & \\
\hline Siempre & 2 & \\
\hline \multicolumn{3}{|c|}{ 7. ¿La Incontinencia Urinaria repercute negativamente en su actividad sexual? } \\
\hline Nunca & $\mathbf{0}$ & \multirow[t]{2}{*}{ puntos } \\
\hline A veces & 1 & \\
\hline
\end{tabular}




\begin{tabular}{|c|c|c|}
\hline Siempre & 2 & \\
\hline \multicolumn{3}{|c|}{ 8. ¿La Incontinencia Urinaria la ha limitado en su vida social (recreación, reuniones)? } \\
\hline Nunca & $\mathbf{0}$ & \multirow[t]{3}{*}{ puntos } \\
\hline A veces & 1 & \\
\hline Siempre & 2 & \\
\hline \multicolumn{3}{|c|}{ 9. ¿La Incontinencia Urinaria la ha limitado y afectado negativamente en su trabajo? } \\
\hline Nunca & $\mathbf{0}$ & \multirow[t]{3}{*}{ puntos } \\
\hline A veces & 1 & \\
\hline Siempre & 2 & \\
\hline \multicolumn{3}{|c|}{ 10. ¿La Incontinencia Urinaria la ha convertido en una persona solitaria y apartada? } \\
\hline Nunca & $\mathbf{0}$ & \multirow[t]{3}{*}{ puntos } \\
\hline A veces & 1 & \\
\hline Siempre & 2 & \\
\hline \multicolumn{3}{|c|}{ 11. ¿La Incontinencia Urinaria ha provocado en usted crisis depresivas o de angustia? } \\
\hline Nunca & $\mathbf{0}$ & \multirow[t]{3}{*}{ puntos } \\
\hline A veces & 1 & \\
\hline Siempre & 2 & \\
\hline \multicolumn{3}{|c|}{ 12. ¿Necesita usted usar pañales o toallas diariamente por su incontinencia urinaria? } \\
\hline Nunca & $\mathbf{0}$ & \multirow[t]{3}{*}{ puntos } \\
\hline A veces & 1 & \\
\hline Siempre & 2 & \\
\hline \multicolumn{3}{|c|}{ 13. ¿Cree usted que la Incontinencia Urinaria tiene tratamiento? } \\
\hline Nunca & $\mathbf{0}$ & \multirow[t]{3}{*}{ puntos } \\
\hline A veces & 1 & \\
\hline Siempre & 2 & \\
\hline \multicolumn{3}{|c|}{$\begin{array}{l}\text { 14. ¿Se sometería usted a alguna modalidad de tratamiento para mejorar y/o curar su Incontinencia } \\
\text { urinaria? }\end{array}$} \\
\hline Nunca & $\mathbf{0}$ & \multirow[t]{3}{*}{ puntos } \\
\hline A veces & 1 & \\
\hline Siempre & 2 & \\
\hline
\end{tabular}

